\title{
INDEX THEORY FOR PERTURBED DIRAC OPERATORS ON MANIFOLDS WITH CONICAL SINGULARITIES
}

\author{
JEFFREY FOX AND PETER HASKELL
}

(Communicated by Palle E. T. Jorgensen)

\begin{abstract}
On an odd-dimensional manifold with isolated conical singularities, we perturb a Dirac operator by a vector bundle endomorphism whose pointwise norm grows in inverse proportion to the distance from the singular set. We give two proofs of an index formula for the resulting Fredholm operator. We mention an application to the index theory of transversally elliptic operators.
\end{abstract}

\section{INTRODUCTION}

In this paper we describe a class of Fredholm perturbed Dirac operators on odd-dimensional manifolds with isolated conical singularities, we prove an index theorem for these operators, and we indicate an application of this index theorem. The analysis of these operators is based on the theory of regular singular operators [BS1]. The perturbations used are invertible in a neighborhood of the singular set. In a neighborhood of the singular set, each perturbations pointwise norm grows in inverse proportion to the distance from the singular set.

Section one of this paper describes the perturbed Dirac operators that we use and establishes their properties. Section two proves an index formula for these operators. A remark at the end of the second section indicates a framework in which this index formula can be applied to the index theory of transversally elliptic operators. We plan to discuss this subject more thoroughly in other papers.

We believe that the significance of this paper lies in noting two roles played by operators of the type we consider: they provide computable index invariants for some transversally elliptic operators; and they establish common ground between index theory on incomplete manifolds and index theory on complete manifolds. Our first interest was in establishing and applying a computable index formula for the perturbed Dirac operators described here. We then noticed

Received by the editors October 22, 1993.

1991 Mathematics Subject Classification. Primary 58G10.

Key words and phrases. Perturbed Dirac operator, regular singular operator, relative index theory, adiabatic limit of reduced eta invariants, transversally elliptic operator.

The first author's work was supported by the National Science Foundation.

The second author's work was supported by the National Science Foundation under Grant No. DMS-9204275. 
similarities with some index results arising on complete manifolds. (Of course we are not the first to notice similarities of this type. The techniques of [B1] and [B2] are based on identifying and using such similarities to prove index theorems on complete manifolds.) To illustrate two points of view on the relationship between index theory on complete manifolds and index theory on incomplete manifolds, we offer two proofs of the index theorem for the perturbed Dirac operators we study. One proof, based on the general form of the index theorem of [BS1], relative index theory, and explicit solution of regular singular differential equations, follows closely the proof appearing in [A] for a class of examples on complete manifolds. The other proof, based on the index theorem of [BS1] and the adiabatic limit calculations of $[\mathrm{BiC}]$, also has analogues in index theory on complete manifolds [BM]. Hence we hope that our paper will play a part in unifying the index theory of Fredholm elliptic operators on complete manifolds and the index theory of such operators on incomplete manifolds. We believe that the presence of a perturbation that is invertible and unbounded at infinity will be central to the strongest forms of such a unified theory. For a perspective on the role played by such a perturbation in the complete case, see [Ga].

\section{Regular Singular Perturbed Dirac operators}

In this section we define and describe the properties of the perturbed Dirac operators that are the subject of this paper.

Let $M$ be the set of smooth points of a compact space with isolated metrically conical singularities. $M$ is an incomplete smooth Riemannian manifold that is divided by a submanifold $N$ into two parts: a compact manifold with boundary, where $N$ is the boundary; and a finite-length metric cone over $N$. This cone equals, for some positive $p,\{(r, n): 0<r \leq p, n \in N\}$ with Riemannian metric $d r \otimes d r+r^{2} g_{N}$. Here $g_{N}$ is a Riemannian metric on $N$. We denote this cone by $C_{0, p}(N) . M$ is the union of these two pieces modulo identification of each point $(p, n)$ in the cone with the corresponding point $n$ in the boundary of the first piece.

Assumption 1.1. Throughout the first three sections of this paper, we assume that $M$ is as above. Moreover we assume that $M$ is an odd-dimensional spin manifold. We fix a spin structure.

Notation 1.2. Let $\mathfrak{D}$ be the Dirac operator defined on sections of the spinor bundle $S \rightarrow M$. Assume for now that the domain of $\mathfrak{D}$ is the set of smooth compactly supported spinors. The domain of $\mathfrak{D}$ will not play an important role in this paper. Let $\widetilde{\mathfrak{D}}$ denote the Dirac operator on the bundle of spinors $\widetilde{S} \rightarrow N$ on $N$.

Remark 1.3 [BS1], [BS2], [C2], [C3], [C4], [Ch1], [Ch2]. Because $M$ is incomplete, $\mathfrak{D}$ may fail to be an essentially self-adjoint operator on the Hilbert space of $L^{2}$ sections of $S$. However, when $\mathfrak{D}$ has self-adjoint extensions, their spectra are similar in many ways to the spectrum of a Dirac operator on a compact manifold without boundary.

Notation 1.4. Let $E$ be a Hermitian vector bundle over $M$. We assume that over the cone $E$ 's structure behaves as the product of $(0, p]$ with the structure of a bundle $\widetilde{E} \rightarrow N$. Thus we fix an identification

$$
\left.E\right|_{C_{0, p}(N)} \cong(0, p] \times \widetilde{E} .
$$


Notation 1.5. Let $\mathfrak{A}$ be a smooth vector bundle endomorphism of $E$. We assume that over $C_{0, p}(N), \mathfrak{A}$ has the form

$$
\mathfrak{A}=r^{-1} \tilde{\mathfrak{A}}
$$

where $\widetilde{\mathfrak{A}}$ is a smooth invertible self-adjoint vector bundle endomorphism of $\widetilde{E}$. Notation 1.6. Choose a metric connection on $E$ that is flat in the radial direction on the cone and that decomposes over the cone into the direct sum of connections on the two subbundles associated with the positive and negative eigenspaces of $\mathfrak{A}$. Using the tensor product connection on $S \otimes E$, we can define a Dirac operator on smooth compactly supported sections of $S \otimes E$. We denote both this operator and its closed extensions by $D$. We denote by $A$ the operator $I \otimes \mathfrak{A}$ on (sections of) $S \otimes E$. Let $\widetilde{S} \rightarrow N$ denote the spinor bundle over $N$. Let $\widetilde{D}$ denote the Dirac operator on sections of $\widetilde{S} \otimes \widetilde{E}$. Let $\widetilde{A}$ denote the operator $I \otimes \widetilde{\mathfrak{A}}$ on sections of the same bundle.

Notation 1.7. Let $\lambda$ denote the operator on sections of $\left.S\right|_{C_{0, p}(N)}$ associated with left Clifford multiplication by the volume form of $N$. (A volume form includes a factor of an appropriate power of $i$. See, e.g., [L].) We orient $N$ so that the tangent vector pointing towards the cone tip followed by the orientation of $N$ agrees with the orientation of $M$. By slight abuse of notation, let $\lambda$ also denote the operator that is really $\lambda \otimes I$ on sections of $S \otimes E$ over the cone.

Lemma 1.8. Let $\tilde{\mathfrak{D}}$ denote the Dirac operator on the bundle of spinors $\widetilde{S}$ over $N$. Under the natural unitary identification of the restriction to the cone of $L^{2}(S)$, respectively $L^{2}(S \otimes E)$, with the Hilbert space of $L^{2}$ functions on $(0, p]$ with values in $L^{2}(\widetilde{\boldsymbol{S}})$, respectively $L^{2}(\widetilde{\boldsymbol{S}} \otimes \widetilde{E}), \mathfrak{D}$ is represented by

$$
i \lambda\left(\partial / \partial r-r^{-1} i \lambda \widetilde{\mathfrak{D}}\right),
$$

and $D \pm i A$ is represented by

$$
i \lambda\left(\partial / \partial r+r^{-1}(-i \lambda \widetilde{D} \pm \lambda \widetilde{A})\right) .
$$

Proof. See [L], which uses calculations of [Ch2].

Lemma 1.9. The operators $\mathfrak{D}$ and $D \pm i A$ are regular singular operators in the sense of [BS1].

Proof. This is an immediate consequence of the preceding lemma once one notes that the Kato-Rellich theorem (see [ReSi]) implies that $i \lambda \widetilde{D} \mp \lambda \widetilde{A}$ is selfadjoint.

Lemma 1.10. For some positive constant $k$ the spectra of the self-adjoint operators $i \lambda \widetilde{D} \pm \lambda k \widetilde{A}$ have empty intersections with $(-1 / 2,1 / 2)$. The set of such $k$ contains an interval that is unbounded on the right.

Proof. $(i \lambda \widetilde{D} \pm \lambda \widetilde{A})^{*}(i \lambda \widetilde{D} \pm \lambda \widetilde{A})=\widetilde{D}^{2}+\widetilde{A}^{2} \pm i[\widetilde{A}, \widetilde{D}]$. The last term is a bounded operator that depends linearly on $\tilde{A}$. (See, e.g., [A, Proposition 1.15].)

Assumption 1.11. Henceforth we always assume that $\mathfrak{A}$ has been chosen so that the spectra of $i \lambda \widetilde{D} \pm \lambda \widetilde{A}$ have empty intersections with $(-1 / 2,1 / 2)$.

Assumption 1.11'. At one point in section 2, we assume that $\mathfrak{A}$ has been chosen so that the spectra of $i \lambda \widetilde{D} \pm \lambda c \widetilde{A}$ have empty intersections with $(-1 / 2,1 / 2)$ for all $c \geq 1$. 
Lemma 1.12 [BS1]. For $\mathfrak{A}$ satisfying Assumption 1.11, the operator $D+i A$, defined originally on the set of smooth compactly supported sections of $S \otimes E$, has a unique closed extension as an operator on $L^{2}(S \otimes E)$. This closed extension is Fredholm. The same assertions are true of $D-i A$.

Notation 1.13. Henceforth we use the notation $D \pm i A$ to refer to the closed extensions mentioned in the preceding lemma.

Lemma 1.14 [BS1], [BS2]. For $\mathfrak{A}$ satisfying Assumption 1.11, $(D+i A)^{*}(D+i A)$ and $(D-i A)^{*}(D-i A)$ have discrete spectra, of finite multiplicity, accumulating only at infinity.

The following corollary is an immediate consequence of this lemma.

Corollary 1.15. The operators $D+i A$ and $D-i A$ are Fredholm.

\section{THE INDEX FORMULA}

In this section we state and give two proofs of an index theorem for operators of the form $D+i A$. One proof starts by observing that the general form of the index theorem of [BS1] and relative index theory imply that the index depends only on the behavior of $D+i A$ on the cone. It thus suffices to calculate explicitly the index of an operator we construct on the product of $N$ with an interval. (The metric on this product causes both ends of the product to be isometric to the cone appearing in $M$.) This proof follows closely the analysis of perturbed Dirac operators on certain complete manifolds that appears in [A]. This proof requires more restrictive conditions on the perturbation $A$ than are needed in the rest of the paper. These conditons can be removed in the context of a $K$-theoretic discussion that will appear in another paper.

The second proof, which works for any perturbation satisfying Assumption $1.11^{\prime}$, proceeds by analyzing the terms that appear in the index theorem of [BS1]. The key step is to analyze the reduced eta invariant by the adiabatic limit technique of $[\mathrm{BiC}]$.

Lemma 2.1. Let $M_{1}$ and $M_{2}$ be manifolds with the properties described for $M$ in section 1. Let $B_{1}$ and $B_{2}$ be operators on $M_{1}$ and $M_{2}$ respectively with the properties described for $D+i A$ in section 1 . Suppose the cone in $M_{1}$ is isometric to the cone in $M_{2}$ by an isometry that extends to an isomorphism of Hermitian vector bundles over the cones and that intertwines the restrictions of $B_{1}$ and $B_{2}$ to the cones. Then the index of $B_{1}$ equals the index of $B_{2}$.

Proof. The index of each of these operators is given by the integral of the standard index form over the manifold plus a contribution arising strictly from the cone [BS1]. The integral of the index form is zero on the odd-dimensional manifold.

Notation 2.2. Let $\widetilde{E}_{+}$, respectively $\widetilde{E}_{-}$, denote the subbundle of $\widetilde{E}$ associated with the positive, respectively negative, eigenspace of $\widetilde{\mathfrak{A}}$. Let $\widetilde{D}_{\widetilde{E}_{+}}$, respectively $\widetilde{D}_{\widetilde{E}_{-}}$, denote the restriction of $\widetilde{D}$ to sections of each of these subbundles. Let $\widetilde{S}=\widetilde{S}^{+} \oplus \widetilde{S}^{-}$denote the spinor bundle, and its decomposition into positive and negative spinor bundles, over $N$. For a Dirac operator on $N$ acting on sections of the tensor product of $\widetilde{S}$ with another bundle, a superscript + denotes the 
part of this operator defined on the tensor product of $\widetilde{S}^{+}$with the other bundle, and the superscript - denotes the adjoint of this operator.

Lemma 2.3. Index $\left(\widetilde{D}_{\widetilde{E}_{+}}^{+}\right)=-\operatorname{index}\left(\widetilde{D}_{\widetilde{E}_{-}}^{+}\right)$.

Proof. The sum of the indices of these Dirac operators equals the index of $\widetilde{D}^{+}$, which is zero by the cobordism invariance of the index.

Remark 2.4. Let $M^{\prime \prime}$ be the disjoint union of two copies of $M$. Let $B^{\prime \prime}$ be the operator on $M^{\prime \prime}$ that equals $D+i A$ on the first copy and $-D-i A$ on the second copy. Because changing the sign of an operator does not change its index, the index of $B^{\prime \prime}$ equals twice the index of $D+i A$.

Definition 2.5. Let $M^{\prime}$ be the product $(-2 p, 2 p) \times N$, and let $E^{\prime}$ be the vector bundle $(-2 p, 2 p) \times \widetilde{E}$, with Hermitian structure, decomposition into positive and negative subbundles, and connection respecting this decomposition all taken from the corresponding structures on $\widetilde{E}$. Orient $M^{\prime}$ by using the standard orientation of the interval followed by the orientation $N$ inherits from $M$. Put a Riemannian metric $d x \otimes d x+f^{2}(x) g_{N}$ on $M^{\prime}$. Here $x$ is the variable from the interval; $g_{N}$ is the Riemannian metric assigned to $N$ earlier; and $f(x)$ is a smooth positive function with values $(x+2 p)$ for $x \in(-2 p,-p)$ and $(2 p-x)$ for $x \in(p, 2 p)$. Let $S^{\prime}$ be the spinor bundle over $M^{\prime}$, and let $D^{\prime}$ be the Dirac operator on sections of $S^{\prime} \otimes E^{\prime}$. Let $A^{\prime}$ be the vector bundle endomorphism of $S^{\prime} \otimes E^{\prime}$ that agrees with $\tilde{A}$ over each $x \in(-2 p, 2 p)$. Let $h(x)$ be a smooth real-valued function that is identically equal to -1 for $x \in(-2 p,-p)$ and identically equal to 1 for $x \in(p, 2 p)$. Let

$$
B^{\prime}=D^{\prime}+(h(x) / f(x)) i A^{\prime} .
$$

Remark 2.6. $\left(B^{\prime}\right)^{*}=D^{\prime}-(h(x) / f(x)) i A^{\prime}$.

Remark 2.7. The reasoning appearing in the statement and proof of Lemma 1.8 allows us to identify $L^{2}\left(S^{\prime} \otimes E^{\prime}\right)$ with $L^{2}\left((-2 p, 2 p), L^{2}(\widetilde{S} \otimes \widetilde{E})\right)$. Then $B^{\prime}$ is represented by

$$
-i \lambda\left(\partial / \partial x+(f(x))^{-1}(i \lambda \widetilde{D}-\lambda h(x) \tilde{A})\right)
$$

$\left(B^{\prime}\right)^{*}$ is represented by

$$
-i \lambda\left(\partial / \partial x+(f(x))^{-1}(i \lambda \widetilde{D}+\lambda h(x) \tilde{A})\right)
$$

Lemma 2.8. Index $\left(B^{\prime}\right)=$ index $\left(B^{\prime \prime}\right)$.

Proof. Apply Lemma 2.1 in the context of the following observations. There is a natural orientation-preserving identification of the cone in the first copy of $M$ in $M^{\prime \prime}$ with the right cone in $M^{\prime}$. There is a natural orientation-reversing identification of the cone in the second copy of $M$ in $M^{\prime \prime}$ with the left cone in $M^{\prime}$. The orientation-reversing identification intertwines the Dirac operator on one cone with the negative of the Dirac operator on the other cone. Also $h(x)$ is negative on the left end of $M^{\prime}$.

Lemma 2.9. Assume that the endomorphism $\tilde{\mathfrak{A}}$ used to define $\tilde{A}$ is a positive multiple of a smooth self-adjoint unitary endomorphism and that $\mathfrak{A}$ satisfies Assumption 1.11. Then

$$
\operatorname{Index}\left(B^{\prime}\right)=\operatorname{index}\left(\widetilde{D}_{\widetilde{E}_{-}}^{+}\right)-\operatorname{index}\left(\widetilde{D}_{\widetilde{E}_{+}}^{+}\right) \text {. }
$$


Proof. Using eigenfunctions of $\widetilde{D}$ to form a basis of $L^{2}(\widetilde{S} \otimes \widetilde{E})$, we see that identifying the kernel of $B^{\prime}$ reduces to solving each of a collection of systems of ordinary differential equations

$$
-i \lambda \partial / \partial x+(f(x))^{-1}(\delta I+i h(x) \alpha I) .
$$

Here $I$ is the $2 \times 2$ identity matrix, and $\lambda$ is the $2 \times 2$ diagonal matrix with 1 in the upper left corner and -1 in the lower right corner. The block structure of the matrices is determined by the decomposition $\widetilde{S}=\widetilde{S}^{+} \oplus \widetilde{S}^{-} . \delta$ is the eigenvalue of $\widetilde{D}$, and $\alpha$ is the value by which $\widetilde{A}$ multiplies the associated eigenvector.

Similarly identifying the kernel of $\left(B^{\prime}\right)^{*}$ reduces to solving a collection of systems of the following form:

$$
-i \lambda \partial / \partial x+(f(x))^{-1}(\delta I-i h(x) \alpha I) .
$$

Because there is an isomorphism between the positive and negative spectral subspaces of the $\widetilde{D}$ associated with changing the sign of an eigenvalue, the difference in the dimensions of the kernels of $B^{\prime}$ and $\left(B^{\prime}\right)^{*}$ equals the difference in the dimensions of those parts of these kernels arising from the kernel of $\widetilde{D}$.

Focusing on the contributions of kernel $(\widetilde{D})$, we can calculate which solutions of the resulting first order regular singular differential equations are $L^{2}$. The kernel of $B^{\prime}$ arises from elements of kernel $\left(\widetilde{D}_{\widetilde{E}_{-}}^{+}\right)$and elements of $\operatorname{kernel}\left(\widetilde{D}_{\widetilde{E}_{+}}^{-}\right)$. The kernel of $\left(B^{\prime}\right)^{*}$ arises from elements of $\operatorname{kernel}\left(\widetilde{D}_{\widetilde{E}_{+}}^{+}\right)$and elements of $\operatorname{kernel}\left(\widetilde{D}_{\widetilde{E}_{-}}^{-}\right)$. It follows that $\operatorname{dim}\left(\operatorname{ker}\left(B^{\prime}\right)\right)-\operatorname{dim}\left(\operatorname{ker}\left(\left(B^{\prime}\right)^{*}\right)\right)=$ $\operatorname{index}\left(\widetilde{D}_{\widetilde{E}_{-}}^{+}\right)-\operatorname{index}\left(\widetilde{D}_{\widetilde{E}_{+}}^{+}\right)$.

Theorem 2.10. Assume that the endomorphism $\tilde{\mathfrak{A}}$ used to define $\tilde{A}$ is a positive multiple of a smooth self-adjoint unitary endomorphism and that $\mathfrak{A}$ satisfies Assumption 1.11. Then

$$
\text { Index }(D+i A)=\operatorname{index}\left(\widetilde{D}_{\widetilde{E}_{-}}^{+}\right) .
$$

Proof. This follows from Remark 2.4 and Lemmas 2.3, 2.8, and 2.9.

The Atiyah-Singer index theorem provides the following corollary.

Corollary 2.11. Assume that the endomorphism $\tilde{\mathfrak{A}}$ used to define $\tilde{A}$ is a positive multiple of a smooth self-adjoint unitary endomorphism and that $\mathfrak{A}$ satisfies Assumption 1.11. Then

$$
\operatorname{Index}(D+i A)=\int_{N} \operatorname{ch}\left(\widetilde{E}_{-}\right) \wedge \hat{A}(N) .
$$

The extra assumptions in the preceding lemma, theorem, and corollary can be removed by a $K$-theoretic discussion. This will appear in another paper. Alternatively we can prove these results without the extra assumption on the perturbation in the following way. (The equality of the right-hand sides of the formulas in Lemma 2.9, Theorem 2.10, and Corollary 2.11 is independent of the extra assumptions on the perturbation. We express the next theorem using one of the three equivalent formulations.) 
Theorem 2.12. For a perturbation satisfying only the assumptions of section one, including Assumption 1.11',

$$
\operatorname{Index}(D+i A)=\operatorname{index}\left(\widetilde{D}_{\widetilde{E}_{-}}^{+}\right) .
$$

Proof. In our setting the index formula of [BS1] states that the index of $D+i A$ equals the sum of three terms: an integral of a standard index form, a term involving the negative of the reduced eta invariant of the operator on $N$, and a term involving residues of this eta invariant. The first term is zero because $M$ is odd-dimensional. The residues vanish by the scaling argument of [B2, proof of Theorem 4.4]. By [BiC] the reduced eta invariant is invariant under multiplying the perturbation by a factor bigger than one (we are in the range where no integer jumps occur), and so its value equals the value of its adiabatic limit. $[\mathrm{BiC}]$ shows that the negative of this limit has the value asserted for the index of $D+i A$.

Remark 2.13. The perturbed Dirac operators considered in this paper are special cases of what might be called in the language of [BS1] first order regular singular perturbed elliptic operators. From our point of view it is important that the perturbation grow (asymptotically) in inverse proportion to the distance from the singular set. Such operators arise in the index theory of transversally elliptic operators in the following way. (See [At], [Sin] for a discussion of transversally elliptic operators.)

Let $X$ be a closed Riemannian manifold on which the compact Lie group $G$ acts by isometries. Let $T$ be a $G$-invariant first order differential operator on $X$ that is elliptic in directions transverse to the $G$-orbits. The index of $T$ is often regarded as a distribution on $G$. There is an equivalent way to view the index of $T$ (although there is no simple direct calculation that translates between the two points of view). The second point of view, often useful in its own right, is to list for every irreducible unitary $G$-representation $\pi$ the value: multiplicity of $\pi$ in $\operatorname{kernel}(T)$ minus multiplicity of $\pi$ in $\operatorname{kernel}\left(T^{*}\right)$. We refer to the value in the list associated to a representation $\pi$ as the $\pi$-index of $T$.

For a given $\pi$, the $\pi$-index of $T$ is equal to the index of a first order regular singular (perturbed) elliptic operator. The constructions that follow are described fully in [BHe2]. For a given $T$ and $\pi$, form the tensor product of the domain and range bundles for $T$ with the bundle $\mathscr{V}_{\pi}^{*} \rightarrow X$. This last bundle is the product of $X$ with a vector space carrying $\pi$ 's contragredient representation. Define the transversally elliptic operator $T \otimes I$ on the sections of the tensor product bundles. The $\pi$-index of $T$ equals the index of the restriction of $T \otimes I$ to the set of $G$-invariant sections of these bundles. This index can be calculated over the dense open subset $X_{0}$ formed by the union of principal $G$-orbits. This index equals the index of an elliptic operator on the (often incomplete) manifold $X_{0} / G$. When the dimension of a $G$-orbit drops, the condition defining transversal ellipticity becomes stricter. Continuity implies that the stricter condition holds in a neighborhood of a smaller orbit. The "extra ellipticity" as one approaches the edge of $X_{0}$ translates into a perturbation of the elliptic operator arising from the transverse directions of $T$ on $X_{0} / G$. The calculations that arise from this point of view are more complicated than may be apparent from this remark. In particular the operators that arise 
can be more complicated, with fewer nice properties, than the perturbed Dirac operators considered in this paper. We plan to address the questions that arise from this point of view in more detail in other papers.

\section{REFERENCES}

[A] N. Anghel, $L^{2}$-index formulae for perturbed Dirac operators, Comm. Math. Phys. 128 (1990), 77-97.

[At] M. F. Atiyah, Elliptic operators and compact groups, Lecture Notes in Math., vol. 401, Springer-Verlag, Berlin, 1974.

[BiC] J.-M. Bismut and J. Cheeger, $\eta$-invariants and their adiabatic limits, J. Amer. Math. Soc. 2 (1989), 33-70.

[BoS] R. Bott and R. Seeley, Some remarks on the paper of Callias, Comm. Math. Phys. 62 (1978), 235-245.

[B1] J. Brüning, $L^{2}$-index theorems on certain complete manifolds, J. Differential Geom. 32 (1990), 491-532.

[B2] _ On $L^{2}$-index theorems for complete manifolds of rank-one type, Duke Math. J. 66 (1992), 257-309.

[BHe1] J. Brüning and E. Heintze, The asymptotic expansion of Minakshisundaram-Pleijel in the equivariant case, Duke Math. J. 51 (1984), 959-980.

[BHe2] _ Representations of compact Lie groups and elliptic operators, Invent. Math. 50 (1979), 169-203.

[BM] J. Brüning and H. Moscovici, $L^{2}$-index for certain Dirac-Schrödinger operators, Duke Math. J. 66 (1992), 311-336.

[BS1] J. Brüning and R. Seeley, An index theorem for first order regular singular operators, Amer. J. Math. 110 (1988), 659-714.

[BS2] , The resolvent expansion for second order regular singular operators, J. Funct. Anal. 73 (1987), 369-429.

[Ca] C. Callias, Axial anomalies and index theorems on open spaces, Comm. Math. Phys. 62 (1978), 213-234.

[C1] J. Cheeger, $\eta$-invariants, the adiabatic approximation and conical singularities, J. Differential Geom. 26 (1987), 175-221.

[C2] - On the Hodge theory of Riemannian pseudomanifolds, Proc. Sympos. Pure Math., vol. 36, Amer. Math. Soc., Providence, RI, 1980, pp. 91-146.

[C3] Spectral geometry of singular Riemannian spaces, J. Differential Geom. 18 (1983), 575-657.

[C4] , On the spectral geometry of spaces with cone-like singularities, Proc. Nat. Acad. Sci. U.S.A. 76 (1979), 2103-2106.

[Ch1] A. Chou, Criteria for self-adjointness of the Dirac operator on pseudomanifolds, Proc. Amer. Math. Soc. 106 (1989), 1107-1116.

[Ch2] - The Dirac operator on spaces with conical singularities and positive scalar curvatures, Trans. Amer. Math. Soc. 289 (1985), 1-40.

[Ga] C. Gajdzinski, $L^{2}$-index for perturbed Dirac operator on odd dimensional open complete manifold, preprint, 1993.

[GLa] M. Gromov and H. B. Lawson, Positive scalar curvature and the Dirac operator on complete Riemannian manifolds, Inst. Hautes Études Sci. Publ. Math. 58 (1983), 83-196.

[LaMi] H. B. Lawson and M.-L. Michelsohn, Spin geometry, Princeton Univ. Press, Princeton, NJ, 1989.

[L] M. Lesch, Deficiency indices for symmetric Dirac operators on manifolds with conic singularities, Topology 32 (1993), 611-623. 
[ReSi] M. Reed and B. Simon, Fourier analysis, self-adjointness, methods of modern mathematical physics, Vol. II, Academic Press, Orlando, FL, 1975.

[Sin] I. M. Singer, Recent applications of index theory for elliptic operators, Proc. Sympos. Pure Math., vol. 23, Amer. Math. Soc., Providence, RI, 1973, pp. 11-31.

Department of Mathematics, University of Colorado, Boulder, Colorado 80309

E-mail address: jfoxCeuclid.colorado.edu

Department of Mathematics, Virginia Polytechnic Institute and State University, BLACKSBURG, VIRGINIA 24061

E-mail address: haskell@math.vt.edu 